\title{
Quasi-periodic pulsations in solar flares: new clues from the Fermi Gamma-Ray Burst Monitor
}

\author{
D. Gruber ${ }^{1}$, P. Lachowicz ${ }^{2}$, E. Bissaldi ${ }^{3}$, M. S. Briggs ${ }^{4}$, V. Connaughton ${ }^{4}$, J. Greiner ${ }^{1}$, A. J. van der Horst ${ }^{4}$, \\ G. Kanbach ${ }^{1}$, A. Rau ${ }^{1}$, P. N. Bhat ${ }^{4}$, R. Diehl ${ }^{1}$, A. von Kienlin ${ }^{1}$, R. M. Kippen ${ }^{5}$, C. A. Meegan ${ }^{6}$, W. S. Paciesas ${ }^{4}$, \\ R. D. Preece ${ }^{4}$, and C. Wilson-Hodge ${ }^{7}$
}

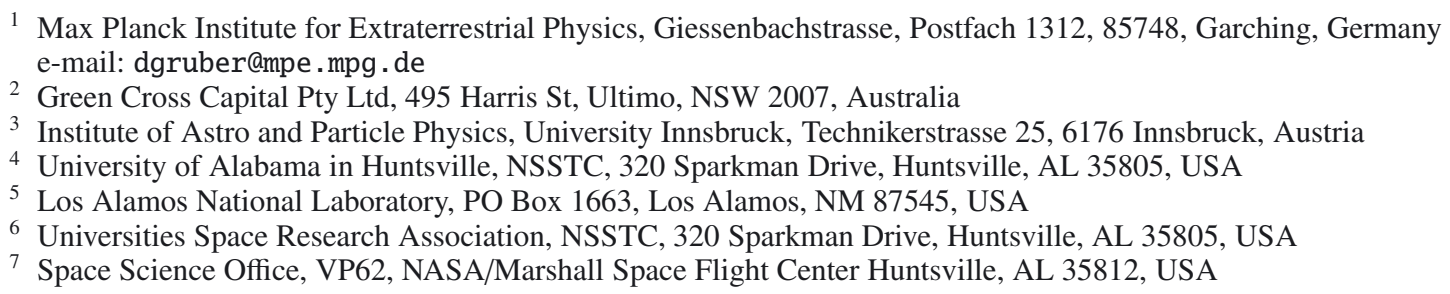

Received 14 April 2011 / Accepted 21 July 2011

\begin{abstract}
Aims. In the past four decades, it has been observed that solar flares display quasi-periodic pulsations (QPPs) from the lowest, i.e. radio, to the highest, i.e. gamma-ray, frequencies in the electromagnetic spectrum. It remains unclear which mechanism creates these QPPs. In this paper, we analyze four bright solar flares that display compelling signatures of quasi-periodic behavior and were observed with the Gamma-Ray Burst Monitor (GBM) onboard the Fermi satellite. Because GBM covers over three decades in energy ( $8 \mathrm{keV}$ to $40 \mathrm{MeV}$ ), it is regarded as a key instrument in our attempt to understand the physical processes that drive solar flares.

Methods. We tested for periodicity in the time series of the solar flares observed by GBM by applying a classical periodogram analysis. However, in contrast to previous authors, we did not detrend the raw light curve before creating the power spectral density (PSD) spectrum. To assess the significance of the frequencies, we used a method that is commonly applied to X-ray binaries and Seyfert galaxies. This technique takes into account the underlying continuum of the PSD, which for all of these sources has a $P(f) \sim f^{-\alpha}$ dependence and is typically labeled red-noise.

Results. We checked the reliability of this technique by applying it to observations of a solar flare that had been observed by the Reuven Ramaty High-Energy Solar Spectroscopic Imager (RHESSI). These data contain, besides any potential periodicity from the Sun, a $4 \mathrm{~s}$ rotational period caused by the rotation of the spacecraft about its axis. We were unable to identify any intrinsic solar quasi-periodic pulsation but we did manage to reproduce the instrumental periodicity. Moreover, with the method adopted here, we do not detect significant QPPs in the four bright solar flares observed by GBM. We stress that for this kind of analyses it is of utmost importance to account appropriately for the red-noise component in the PSD of these astrophysical sources.
\end{abstract}

Key words. Sun: flares - methods: statistical - methods: data analysis - methods: observational

\section{Introduction}

Over the past 40 years, quasi-periodic pulsations (QPP) in solar flares have been reported from observations across the electromagnetic spectrum, i.e. from radio waves to the highly energetic gamma-rays, on timescales from sub-second intervals up to several minutes (e.g. Parks \& Winckler 1969; Ofman \& Sui 2006; Li \& Gan 2008; Nakariakov \& Melnikov 2009; Nakariakov et al. 2010a). While there seems to be an overwhelming amount of observational data, the underlying physical mechanism that could generate such QPPs still remains a mystery. Possible processes that are considered are modulation of electron dynamics by magnetohydrodynamic (MHD) oscillations (Zaitsev \& Stepanov 1982), periodic triggering of energy releases by MHD waves (Foullon et al. 2005; Nakariakov et al. 2006), MHD flow overstabilities (Ofman \& Sui 2006), and oscillatory regimes of magnetic reconnection (Kliem et al. 2000).

In this paper, we present time series and periodogram analyses of four solar flares that display compelling quasi-periodic behavior in their light curves. All of these solar flares were observed by the Fermi Gamma-Ray Burst Monitor (GBM, Meegan et al. 2009). GBM is one of the instruments onboard the Fermi Gamma-Ray Space Telescope (Atwood et al. 2009) launched on June 11, 2008. Specifically designed for gammaray burst (GRB) studies, GBM observes the whole unocculted sky with a total of 12 thallium-activated sodium iodide $(\mathrm{NaI}(\mathrm{Tl}))$ scintillation detectors covering the energy range from $8 \mathrm{keV}$ to $1 \mathrm{MeV}$ and two bismuth germanate scintillation detectors (BGO) sensitive to energies between $150 \mathrm{keV}$ and $40 \mathrm{MeV}$ (Meegan et al. 2009). Therefore, GBM offers superb capabilities for the analyses of not only GRBs but solar flares as well.

The analysis and interpretation of the power spectral density (PSD) of solar flares is, in general, difficult. A variety of astrophysical sources (such as X-ray binaries, Seyfert galaxies (e.g. Lawrence et al. 1987; Markowitz et al. 2003) and GRBs (Ukwatta et al. 2009; Cenko et al. 2010)), show erratic, aperiodic brightness changes. Solar flares exhibit similar aperiodic variations with the general time profile being a sharp impulsive phase followed by a slower decay phase. Solar flares, together with 
many other astrophysical sources, thus have very steep power spectra in the low-frequency region. This type of variability is known as red-noise (e.g. Groth 1975; Deeter \& Boynton 1982; Israel \& Stella 1996; Vaughan 2005). When determining the significance of possible periodicities in the PSD, the red-noise has to be accounted for in order not to severely overestimate the significance of identified frequencies (Lachowicz et al. 2009). In this paper, we account for the red-noise properties when performing a periodogram analysis.

This paper is organized as follows. In Sect. 2, we briefly present the methodology of the time-series analysis. We provide an overview of the red-noise properties in astrophysical sources and demonstrate the importance of the red-noise when estimating significances. In Sect. 3, we present the light curves and periodograms of solar flares that were observed by GBM. Finally, in Sect. 4, we summarize our study and present our conclusions.

\section{Analysis of data governed by red-noise}

As already pointed out by Mandelbrot \& Wallis (1969) and Press (1978), the human eye has a tendency to identify periodicities from purely random time series, i.e. where sinusoidal variations are not statistically real. According to Press (1978), the strongest eye-apparent period in (actually non-periodic) data will be about one-third the length of the data sample. According to these authors "three-cycle" quasiperiods should be taken with a grain of salt.

Solar flares fall into the group of astrophysical sources whose red-noise is important. Red-noise has nothing to do with measurement errors or the systematics of the detectors, which are also called noise. Red-noise is an intrinsic property of the observed source and is due to erratic, aperiodic brightness changes. In contrast to white noise, which displays a flat spectrum in a PSD, i.e. is power independent of frequency, red-noise is characterized by a power law of the form of $P=N f^{-\alpha}$. As a first order approximation, red-noise is the realization of a linear stochastic and weakly non-stationary process. This red-noise component makes the interpretation of the significance of a peak in the PSD more complex.

One way to estimate the significance of induced frequencies on top of an underlying red-noise continuum in a PSD, was presented by Vaughan (2005).

In short, Vaughan suggests calculating the periodogram normalized so that the units of power are $(\mathrm{rms} / \mathrm{mean})^{2} \mathrm{~Hz}^{-1}$ (e.g. Schuster 1898; Press \& Rybicki 1989). Then, the periodogram is converted to log-space in both frequency and power. For such a log-periodogram, one can then clearly identify the power-law component in the low-frequency range and a "cutoff" where white noise or an additional noise component takes over (e.g. Ukwatta et al. 2009). One can easily determine the power-law parameters by fitting a linear function to the low-frequency periodogram bins using the least squares method. In this paper, the method of Vaughan (2005) was slightly modified in that we use a broken power law (BPL) to fit the PSD instead of a single power law.

\subsection{Red-noise simulation}

It is common practice (Inglis et al. 2008; Nakariakov et al. 2010a) to suppress the low-frequency component by de-trending the light curves of solar flares. This can be achieved by smoothing the light curve with a moving average or by applying a Gaussian filter and performing the periodogram analysis on the
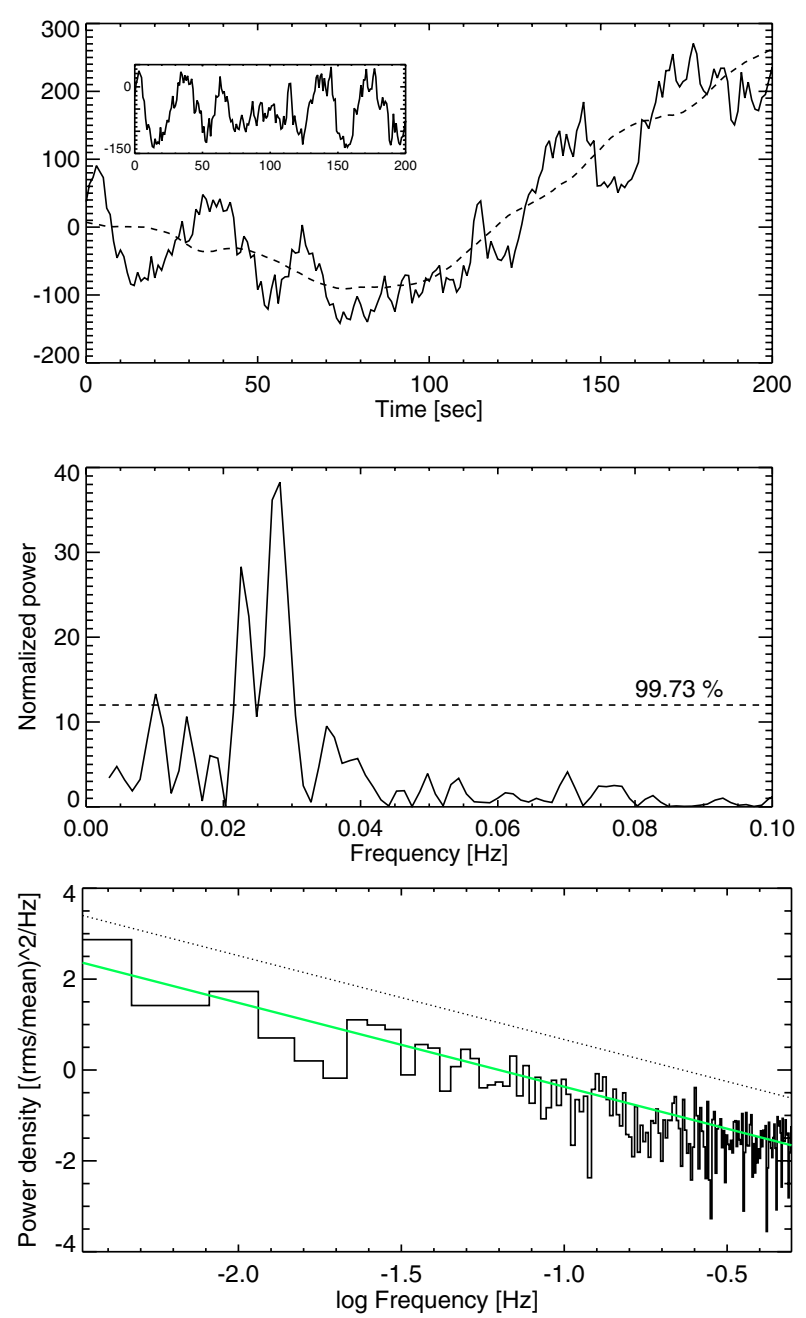

Fig. 1. Upper panel: synthetic red-noise time series with a power law index of $\alpha=-1.8$. Simple (boxcar) moving average is overplotted (dashed line). The inset presents the residual signal. Middle panel: Lomb-Scargle Periodogram of the residual light curve. The $3 \sigma$ CL has been denoted by a dashed line. Lower panel: PSD with the best fit power law (solid green line) and the $3 \sigma$ significance level (dotted line).

residual emission, i.e. the smoothed version is subtracted from the original data-set. This can give rise to misleading results as we show in the following.

By randomizing the phase as well as the amplitudes, Timmer \& Koenig (1995) introduced an algorithm to generate a purely random time series that displays a $1 / f^{\alpha}$ dependence in the PSD. We created a time series consisting of 200 data points, evenly spaced by $1 \mathrm{~s}$ with a $f^{-1.8}$ periodogram shape. From this light curve, we subtracted a simple moving average of $50 \mathrm{~s}$ (see Fig. 1). We then calculated the PSD (first described by Lomb 1976; Scargle 1982, then refined by Press \& Rybicki 1989) of the residuals. The result is remarkable. Although we started with a purely random, red-noise dominated time-series, we obtain a PSD with three frequencies whose power exceeds the $3 \sigma$ confidence limit (calculated according to Scargle 1982). This approach in signal processing clearly returns false-positive frequencies with periods of $P \approx 33 \mathrm{~s}, P \approx 55 \mathrm{~s}$, and $P \approx 100 \mathrm{~s}$, respectively. However, the spectral peaks remain well below the $3 \sigma$ threshold if the periodogram is calculated using the method introduced by Vaughan (2005). 
Therefore, as shown by the example above, we strongly discourage subtracting smoothed versions of raw light curves when looking for intrinsic frequencies in the red-noise dominated PSD.

\subsection{Detour: $Q P P s$ in GRBs}

The same procedure of light curve reprocessing with a subsequent PSD analysis has also been applied to GRBs. These events are the most luminous flashes of $\gamma$-rays known to mankind, believed to originate from highly relativistic outflows from a compact source with Lorentz factors $\Gamma>100$. In 2009, the SwiftBAT satellite (Gehrels et al. 2004) observed GRB 090709A (Morris et al. 2009). Soon after this detection, Markwardt et al. (2009) claimed that the GRB had displayed very unusual behavior, not observed in any other GRB so far, namely a QPP-like behavior with a periodicity of $P \approx 8.1 \mathrm{~s}$ at the $12 \sigma$ level of significance. This QPP was subsequently confirmed and found to be in phase with the data of the Anti-Coincidence System (ACS) of the spectrometer SPI on board the INTEGRAL satellite (Götz et al. 2009), the Konus-WIND instrument (Golenetskii et al. 2009), and the Suzaku Wide-band All-sky Monitor (WAM) (Ohno et al. 2009). These latter instruments operate in the energy ranges $80 \mathrm{keV}-10 \mathrm{MeV}, 20 \mathrm{keV}-1150 \mathrm{keV}$, and $50 \mathrm{keV}-5 \mathrm{MeV}$, respectively. In contrast Swift-BAT, is sensitive to the energy range $15 \mathrm{keV}-150 \mathrm{keV}$.

However, soon thereafter, Cenko et al. (2010) showed that the interpretation of this QPP depends strongly on the assumptions made about the underlying continuum. If the latter is accounted for, the significance of the claimed periodicity drops below the $3 \sigma$ confidence limit. This analysis was independently repeated by Iwakiri et al. (2010) and de Luca et al. (2010), who also took into account the red-noise component in the PSD, and only found a marginally significant periodicity at the $3 \sigma$ confidence limit.

In conclusion to this detour, we emphasize once more the importance of accounting for the red-noise component in the PSD. In addition, we highlight that a potential quasi-periodic signal is not necessarily significant even if it is identified in several instruments with different (but overlapping) energy ranges and observed to be in phase across these bands.

\subsection{Method testing}

The Reuven Ramaty High-Energy Solar Spectroscopic Imager (RHESSI, Lin et al. 2002) rotates about its spin axis which is always directed towards the Sun. The period of this rotation is $P \approx 4 \mathrm{~s}$ and a PSD analysis of the RHESSI light curves is expected to detect this instrumental signal. We applied the Vaughan (2005) test to a solar flare that was observed by RHESSI on January 1st, 2005 and where QPPs have been reported (Nakariakov et al. 2010a). This solar flare peaked at 00:31 UT at a GOES level X1.7, from the NOAA active region 10715 located on disk at N03E47. We used RHESSI data in the energy range $50 \mathrm{keV}-100 \mathrm{keV}$ and a fine time resolution of $0.1 \mathrm{~s}$ (see upper panel of Fig. 2) and performed two periodogram analyses on this light curve in the range between $1660 \mathrm{~s}$ and $1820 \mathrm{~s}$. The first periodogram analysis was performed using the classical approach introduced by Lomb (1976) and Scargle (1982). As commonly performed in the past (e.g. Inglis \& Nakariakov 2009), a periodogram was calculated on the residual emission after a simple moving average of $50 \mathrm{~s}$ was subtracted from the raw data (see middle panel of Fig. 2). With
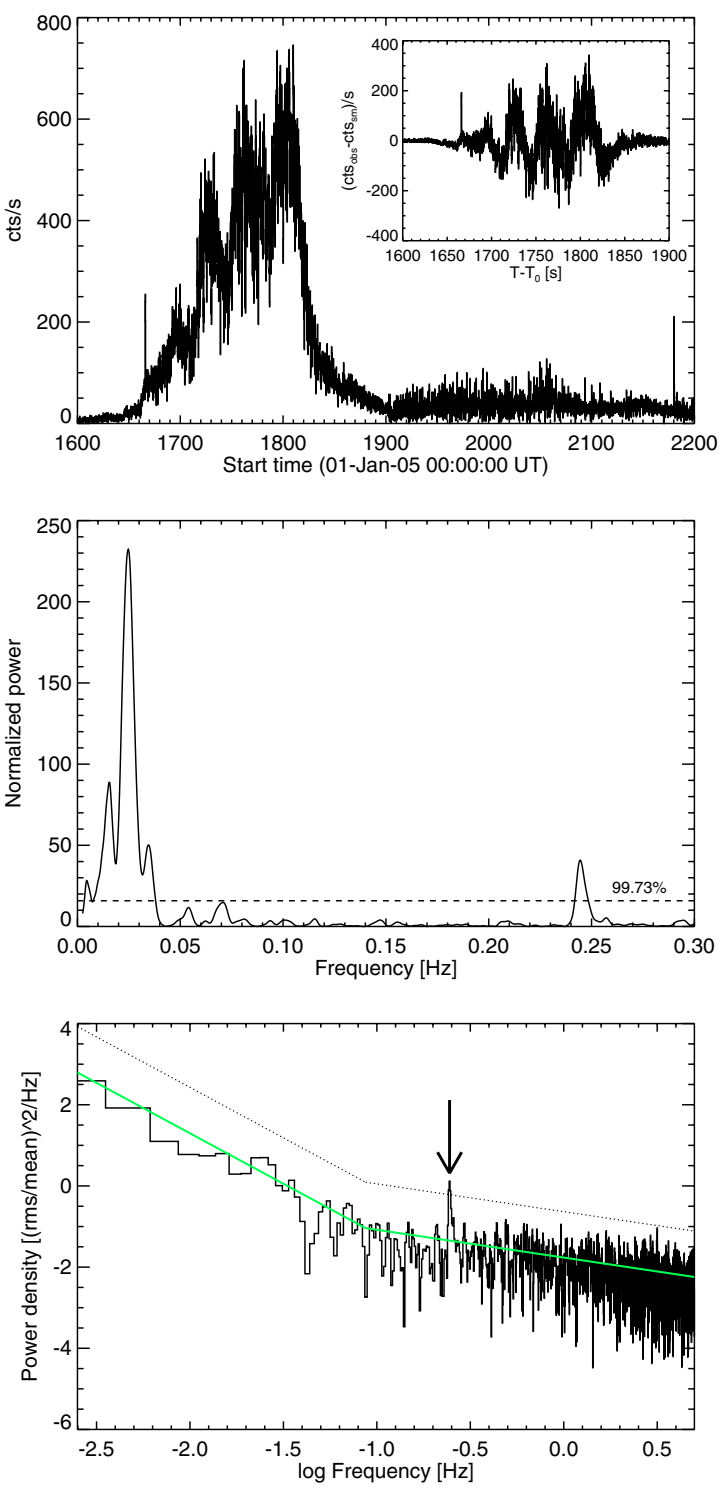

Fig. 2. Upper panel: summed and background subtracted light curve of the solar flare observed by RHESSI on 2005 January. The inset shows the residual emission after subtracting a simple (boxcar) moving average. Middle panel: periodogram analysis performed on the residual emission. Lower panel: PSD with best fit broken power-law (solid green line) and the $3 \sigma$ significance level (dotted line) indicated. The arrow points to the significant frequency at $f \approx 0.244 \mathrm{~Hz}$.

this method, several peaks were found above the $3 \sigma$ threshold. The peak with the highest value of normalized power is located at $f \sim 0.025 \mathrm{~Hz}$, corresponding to the periodicity reported by Nakariakov et al. (2010a). Another peak worth mentioning is located at $f \sim 0.24 \mathrm{~Hz}$, which is the expected rotational frequency of RHESSI around its spin axis.

As we now demonstrate, the significance of the peak at $f \sim 0.025 \mathrm{~Hz}$ is highly overestimated by the latter method. We show a PSD that was calculated using the raw and undetrended light curve applying the technique by Vaughan (2005) in the bottom panel of Fig. 2. In analogy to the Lomb and Scargle periodogram analysis, we found a significant spectral feature at $\approx 0.24 \mathrm{~Hz}$, which is the expected rotation period of the RHESSI instrument. However, this PSD is lacking any other frequency above the $3 \sigma$ confidence limit. The discrepancy between 

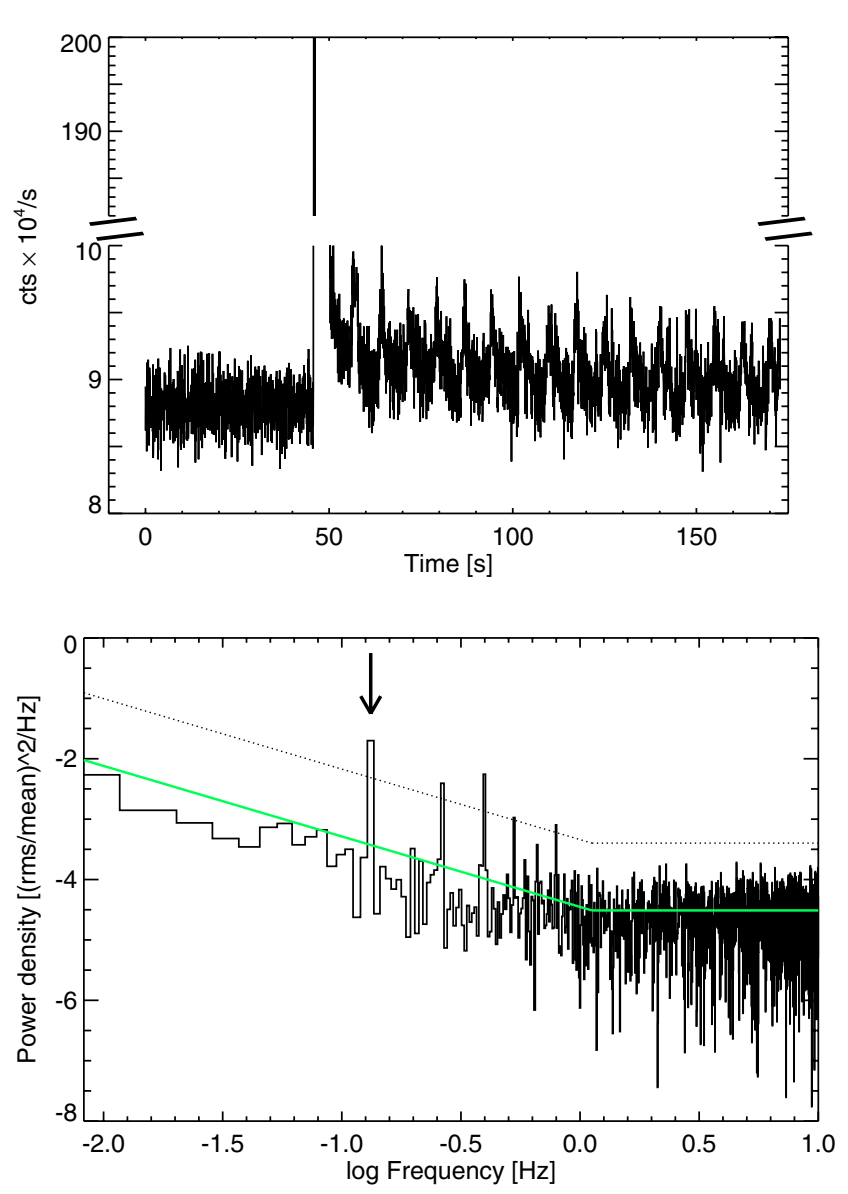

Fig. 3. Upper panel: light curve of the giant flare of SGR 1806-20 observed by SPI-ACS. Lower panel: PSD of the SGR 1806-20 light curve. Best fit broken power-law to the PSD continuum (solid green line) and the $3 \sigma$ significance level (dotted line) are indicated. The arrow indicates the recovered rotation period of $P \approx 7.56 \mathrm{~s}$. Other peaks above the $3 \sigma$ levels are harmonics.

the two methods is easily explained. Firstly, the whole raw light curve was used without performing any artificially detrending beforehand. Secondly, the method of Lomb and Scargle assumes a white noise continuum and does not take into consideration the red noise component of the solar flare. However, the latter is taken into account by the method of Vaughan. In conclusion, we could not confirm the reported QPP in this solar flare. We are confident that this latter method can be used for the subsequent analysis because we believe that it is more appropriate for sources that are dominated by red-noise and for which the inherent rotational frequency of the instrument is found to be significant.

An additional check was performed using SPI-ACS data of the outburst of the soft gamma repeater SGR 1806-20 observed on 2004 December 27 in the energy range from $80 \mathrm{keV}$ to $8 \mathrm{MeV}$ (Mereghetti et al. 2005). SGR 1806-20 is known to have a rotational period of $7.56 \mathrm{~s}$ and the methodology adopted here should be able to recover this periodicity. We removed the very bright initial pulse and focused on the emission from $\approx 50 \mathrm{~s}$ to $175 \mathrm{~s}$ (see Fig. 3). We unambiguously recover the main pulsation pe$\operatorname{riod}(P \approx 7.56 \mathrm{~s})$ together with the first, second, third, and fifth harmonic.

We conclude that the methodology applied here is appropriate and reliable for the following analysis.

\section{Solar flares observed by GBM}

\subsection{Solar flare on February 24, 2011 at 07:29:20.71 UT}

For the analysis, we use CSPEC and CTIME data of detectors $\mathrm{NaI} 3, \mathrm{NaI} 4$, and $\mathrm{NaI} 5$ with a time resolution of $1.024 \mathrm{~s}(4.096 \mathrm{~s}$ pre-trigger) and $0.064 \mathrm{~s}(0.256 \mathrm{~s}$ pre-trigger $)$, respectively. In the energy range from $50 \mathrm{keV}$ to $1 \mathrm{MeV}$, this solar flare lasted for about $500 \mathrm{~s}$. The light curve (Fig. 4) consists of several peaks and a compellingly looking quasi-periodic behavior lasting until $\approx 500 \mathrm{~s}$. After de-trending the raw light curve with a simple moving average $(50 \mathrm{~s})$, the QPP pattern becomes more visible (see inset of Fig. 4). Applying a standard periodogram analysis (Lomb 1976; Scargle 1982) to the detrended light curve, several peaks are above the $3 \sigma$ confidence limit as can be seen in the middle panel of Fig. 4.

However, applying the previously introduced method (Vaughan 2005) to the original light curve, thus taking into account the red-noise properties of the source we did not find any significant QPP during the solar flare life-time, as pointed out in the lower panel of Fig. 4. We performed this analysis making use of the CTIME data with a time resolution of $64 \mathrm{~ms}$. The PSD was calculated for the signal spanning from $\left(T_{0}-10\right) \mathrm{s}$ to $\left(T_{0}+600 \mathrm{~s}\right)$, where $T_{0}$ denotes the time of trigger.

\subsection{Solar flare on June 12,2010 at 00:55:05 UT}

For the analysis, we again use CSPEC and CTIME data of detectors $\mathrm{NaI} 0$ through $\mathrm{NaI} 5$. The count rate did not increase significantly during the first $30 \mathrm{~s}$ in the $50 \mathrm{keV}$ to $1 \mathrm{MeV}$ energy range. After this time, there is a sharp increase in the flare brightness, which then decays again very rapidly after $60 \mathrm{~s}$. The whole duration of the solar flare in this energy range is approximately $120 \mathrm{~s}$. Overlaid on top of the observed light curve, one can again clearly identify a QPP behavior with a period of $\sim 15 \mathrm{~s}$ (see upper panel of Fig. 5). This periodicity appears to be quite significant when applying a standard Lomb-Scargle periodogram (see middle panel of Fig. 5) to the detrended light curve. The smoothing length was $15 \mathrm{~s}$.

However, the PSD, which we computed using CTIME data with a time resolution of $64 \mathrm{~ms}$ in the interval from $\left(T_{0}-25\right) \mathrm{s}$ to $\left(T_{0}+150\right) \mathrm{s}$ and applying the method of to Vaughan (2005), does not display any significant periodicity.

\subsection{Solar flare on March 15, 2011 at 00:21:15.69 UT}

For this analysis, we adopt CTIME data of detectors NaI 0 through $\mathrm{NaI} 5$ in an energy range covering $50 \mathrm{keV}$ to $1 \mathrm{MeV}$ with a time resolution of $0.256 \mathrm{~s}$. The total duration of the solar flare in this energy range is approximately $50 \mathrm{~s}$ (see Fig. 6). For the Lomb-Scargle periodogram analysis (see middle panel of Fig. 6), the light curve was detrended with a simple moving average of $5 \mathrm{~s}$. After repeating the PSD analysis of the original data set between $\left(T_{0}-30\right) \mathrm{s}$ and $\left(T_{0}+100\right) \mathrm{s}$ using the method of Vaughan (2005), we did not find any significant QPP.

\subsection{Solar flare on March 14, 2011 at 19:50:17.3 UT}

For the analysis, we use the same data type, energy range, and time resolution as for solar flare \#4 in Sect. 3.3. The total duration of the solar flare is approximately $150 \mathrm{~s}$ (see Fig. 7). In contrast to the Lomb-Scargle periodogram, which identifies a significant periodicity in the detrended light curve (smoothing length of $10 \mathrm{~s}$ ), the PSD, determined for the time interval 

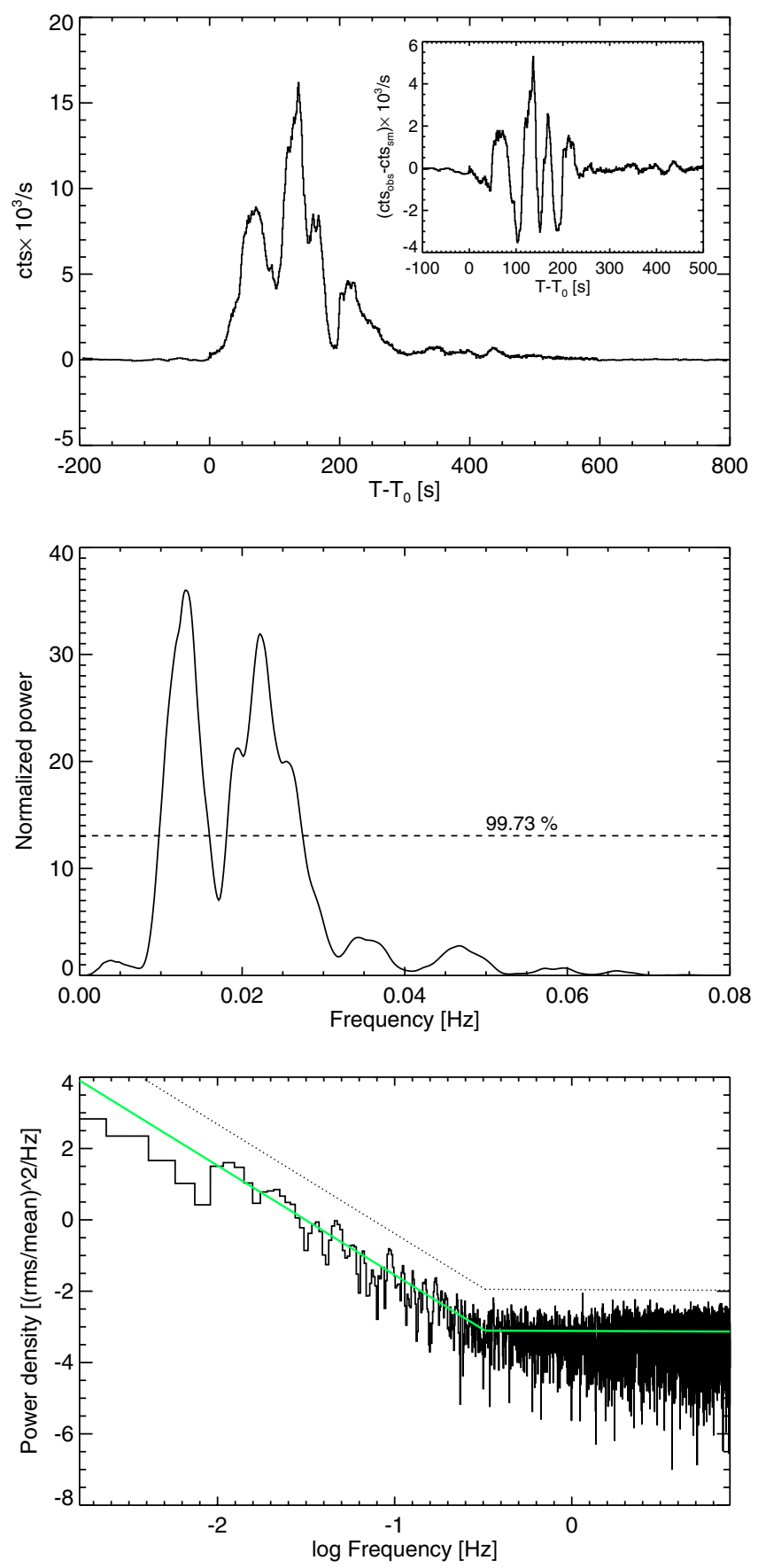

Fig. 4. Upper panel: summed and background subtracted light curve of the solar flare observed by GBM on 2011 February 24. The inset shows the residual emission after subtracting a simple (boxcar) moving average. Middle panel: periodogram analysis performed on the residual emission. Two peaks are above the $3 \sigma$ confidence limit (dashed line). Lower panel: PSD of the same solar flare. Best fit broken power-law to the PSD continuum (solid green line) and the $3 \sigma$ significance level (dotted line) are indicated.

between $\left(T_{0}-25\right) \mathrm{s}$ and $\left(T_{0}+150\right) \mathrm{s}$ using the method of Vaughan (2005), does not identify any significant QPP.

\section{Summary and conclusions}

We have analyzed light curves of five solar flares observed by both GBM and RHESSI. We tested the data for the presence and
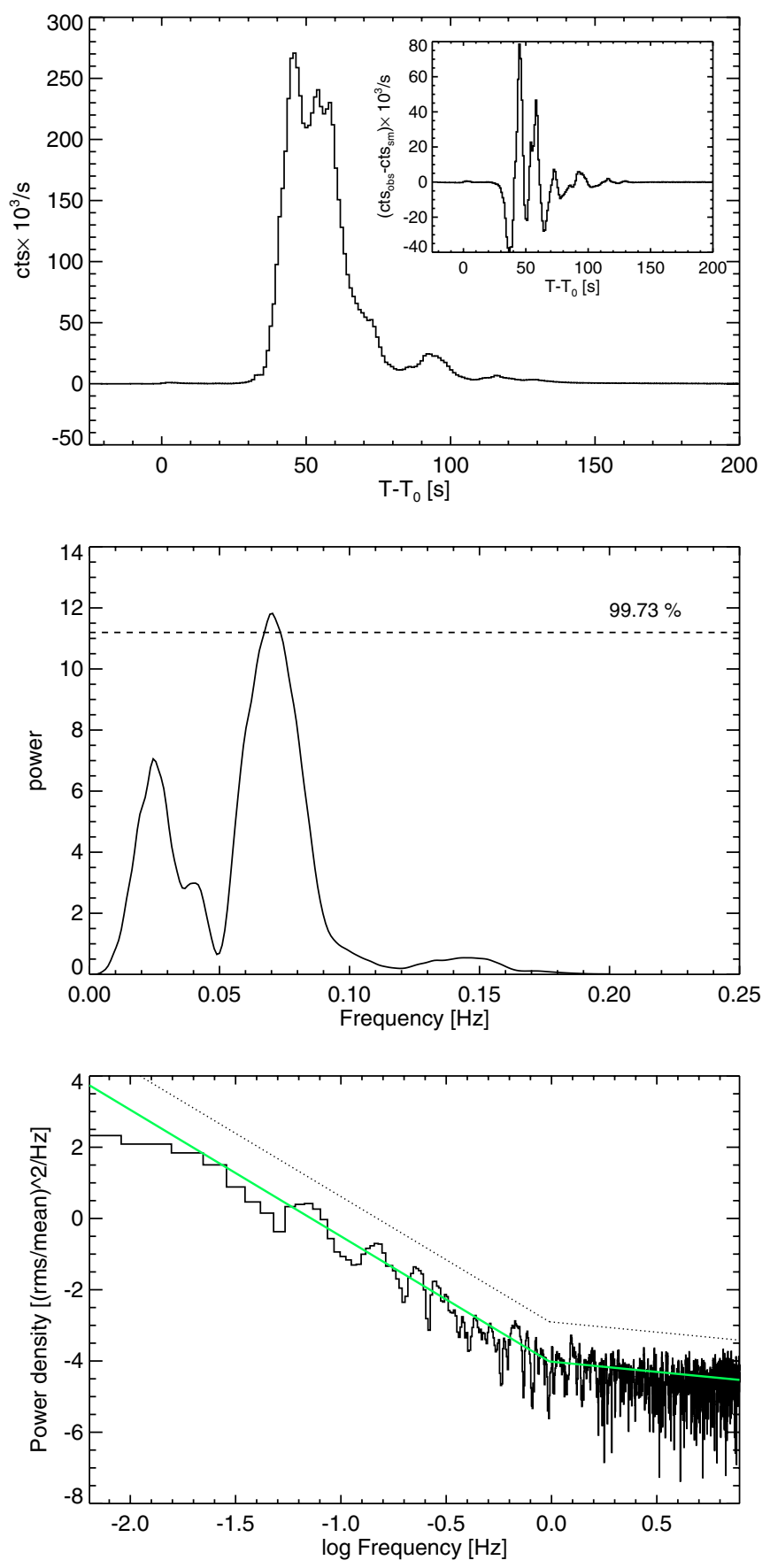

Fig. 5. Upper panel: same as Fig. 4 for the solar flare observed on 2010 June 12. Middle panel: same as Fig. 4. Lower panel: same as Fig. 4.

significance of QPPs accounting for the overall shape of the PSD by applying the method introduced by Vaughan (2005). First of all, this technique was validated and tested by applying it to raw data of the RHESSI satellite. Any RHESSI light curve has an inherent period caused by the rotation of the spacecraft about its axis. With the method adopted here, we successfully retrieved this well-known $4 \mathrm{~s}$ period. However, we were unable to confirm the previously reported QPP of $40 \mathrm{~s}$ in the very same solar flare (Nakariakov et al. 2010a). An additional check was performed by applying the method to SPI-ACS data of the giant flare of the well-known SGR 1806-20. This magnetar has a rotation period of $7.56 \mathrm{~s}$, which, together with several harmonics, could be recovered unambiguously with the procedure presented 

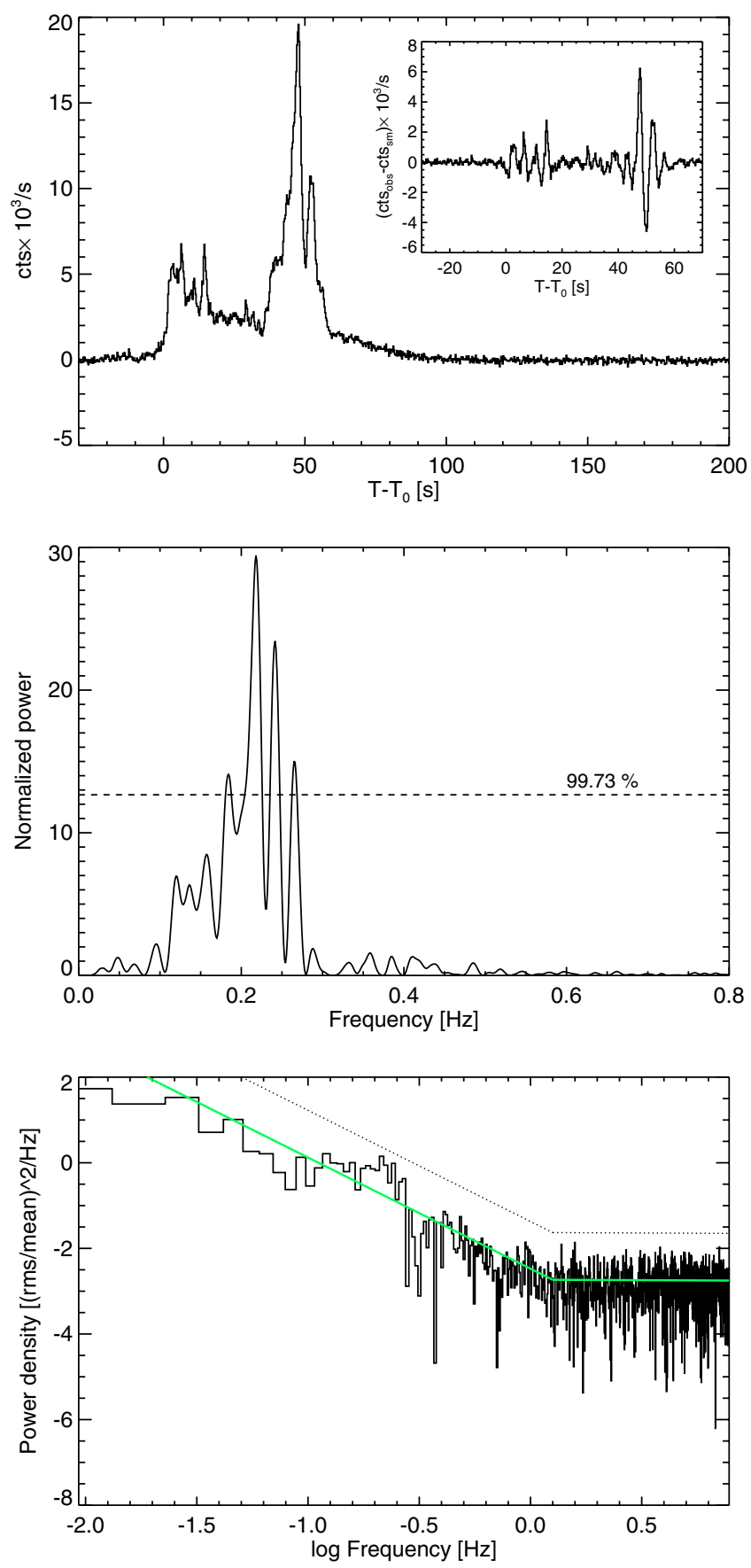

Fig. 6. Upper panel: same as Fig. 4 for the solar flare observed on 2011 March 15. Middle panel: same as Fig. 4. Lower panel: same as Fig. 4.

here. These two tests gave us confidence that the method is appropriate to test the significance of QPPs in red-noise-dominated solar-flare time series.

The routine was then applied to four solar flares observed by GBM . Although all of these solar flares displayed apparently clear quasi-periodic features in their detrended light curves, none of these were significant $(>3 \sigma)$. Previous authors (e.g. Inglis et al. 2008; Inglis \& Nakariakov 2009; Zimovets \& Struminsky 2010; Nakariakov et al. 2010a) who claimed a significant QPP detection, applied a standard Lomb-Scargle analysis to detrended solar-flare light curves. Our investigation of this method suggests that the power in the low frequency range is being artificially suppressed, which can lead to misleading values
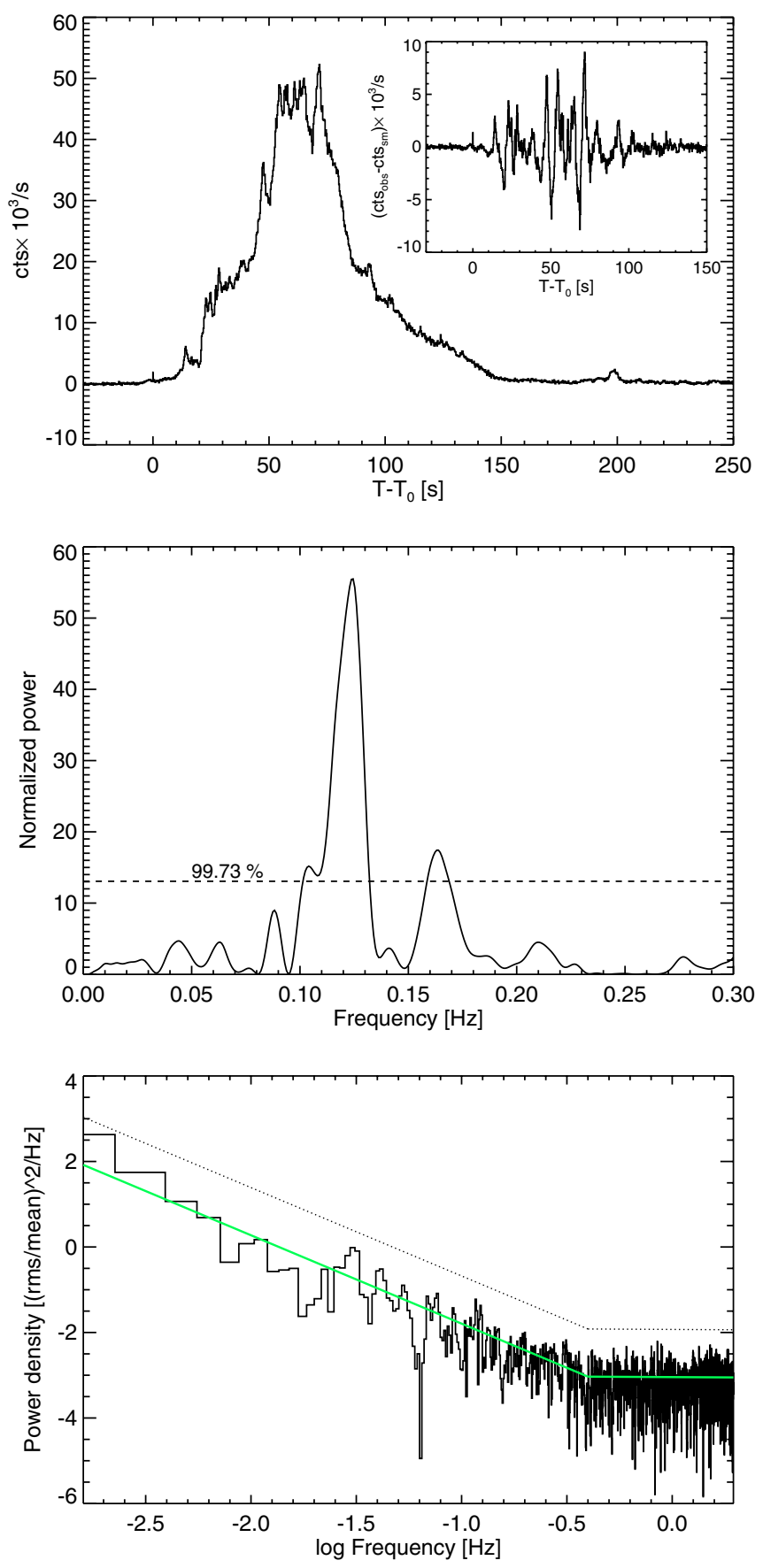

Fig. 7. Upper panel: same as Fig. 4 for the solar flare observed on 2011 March 14. Middle panel: same as Fig. 4. Lower panel: same as Fig. 4.

of the significances of features in the PSD. A periodogram analysis should always be performed on the raw and undetrended light curve as done here.

We emphasize once again that not only solar flares but many astrophysical sources (X-ray binaries, Seyfert galaxies, GRBs) have steep power spectra in the low-frequency range. These power spectra ensure that a periodogram analysis is not trivial, the interpretation of a peak in a PSD is more complex than usual, and that the estimate of the peak's significance is important. Again we emphasize that red-noise is an intrinsic source property. In other words, having shown that the variations in the corresponding solar flares are not quasi-periodic at the $3 \sigma$ level does not mean that these variations are not real. The corresponding 
flux changes are sometimes dramatic, reaching a factor of a few within a few tens of seconds. In addition, these variations occur in phase at different X-ray to gamma-ray energies, and other flares have been observed to also occur in phase with microwave radio emission (e.g. Nakariakov et al. 2010a; Foullon et al. 2010).

While we consider it unnecessary to invoke oscillatory regimes of plasma instabilities (Nakariakov et al. 2010b; Reznikova \& Shibasaki 2011), we note that the physics of these variations is certainly interesting and worth further study.

\section{References}

Atwood, W. B., Abdo, A. A., Ackermann, M., et al. 2009, ApJ, 697, 1071

Cenko, S. B., Butler, N. R., Ofek, E. O., et al. 2010, AJ, 140, 224

de Luca, A., Esposito, P., Israel, G. L., et al. 2010, MNRAS, 402, 1870

Deeter, J. E., \& Boynton, P. E. 1982, ApJ, 261, 337

Foullon, C., Verwichte, E., Nakariakov, V. M., \& Fletcher, L. 2005, A\&A, 440, L59

Foullon, C., Fletcher, L., Hannah, I. G., et al. 2010, ApJ, 719, 151

Gehrels, N., Chincarini, G., Giommi, P., et al. 2004, ApJ, 611, 1005

Golenetskii, S., Aptekar, R., Mazets, E., et al. 2009, GRB Coordinates Network, 9647,1

Götz, D., Mereghetti, S., von Kienlin, A., \& Beck, M. 2009, GRB Coordinates Network, 9649, 1

Groth, E. J. 1975, ApJS, 29, 453

Inglis, A. R., \& Nakariakov, V. M. 2009, A\&A, 493, 259

Inglis, A. R., Nakariakov, V. M., \& Melnikov, V. F. 2008, A\&A, 487, 1147

Israel, G. L., \& Stella, L. 1996, ApJ, 468, 369

Iwakiri, W., Ohno, M., Kamae, T., et al. 2010, in AIP Conf. Ser. 1279, ed. N. Kawai, \& S. Nagataki, 89

Kliem, B., Karlický, M., \& Benz, A. O. 2000, A\&A, 360, 715
Lachowicz, P., Gupta, A. C., Gaur, H., \& Wiita, P. J. 2009, A\&A, 506, L17 Lawrence, A., Watson, M. G., Pounds, K. A., \& Elvis, M. 1987, Nature, 325, 694

Li, Y. P., \& Gan, W. Q. 2008, Sol. Phys., 247, 77

Lin, R. P., Dennis, B. R., Hurford, G. J., et al. 2002, Sol. Phys., 210, 3

Lomb, N. R. 1976, Ap\&SS, 39, 447

Mandelbrot, B. B., \& Wallis, J. R. 1969, Water Resources Research, 5, 1, 228

Markowitz, A., Edelson, R., Vaughan, S., et al. 2003, ApJ, 593, 96

Markwardt, C. B., Gavriil, F. P., Palmer, D. M., Baumgartner, W. H., \& Barthelmy, S. D. 2009, GRB Coordinates Network, 9645, 1

Meegan, C., Lichti, G., Bhat, P. N., et al. 2009, ApJ, 702, 791

Mereghetti, S., Götz, D., von Kienlin, A., et al. 2005, ApJ, 624, L105

Morris, D. C., Beardmore, A. P., Evans, P. A., et al. 2009, GRB Coordinates Network, 9625, 1

Nakariakov, V. M., \& Melnikov, V. F. 2009, Space Sci. Rev., 149, 119

Nakariakov, V. M., Foullon, C., Verwichte, E., \& Young, N. P. 2006, A\&A, 452, 343

Nakariakov, V. M., Foullon, C., Myagkova, I. N., \& Inglis, A. R. 2010a, ApJ, 708, L47

Nakariakov, V. M., Inglis, A. R., Zimovets, I. V., et al. 2010b, Plasma Physics and Controlled Fusion, 52, 124009

Ofman, L., \& Sui, L. 2006, ApJ, 644, L149

Ohno, M., Iwakiri, W., Suzuki, M., et al. 2009, GRB Coordinates Network, 9653, 1

Parks, G. K., \& Winckler, J. R. 1969, ApJ, 155, L117

Press, W. H. 1978, Comm. Astrophys., 7, 103

Press, W. H., \& Rybicki, G. B. 1989, ApJ, 338, 277

Reznikova, V. E., \& Shibasaki, K. 2011, A\&A, 525, A112

Scargle, J. D. 1982, ApJ, 263, 835

Schuster, A. 1898, J. Geophys. Res., 3, 13

Timmer, J., \& Koenig, M. 1995, A\&A, 300, 707

Ukwatta, T. N., et al. 2009 [arXiv:0906. 3193]

Vaughan, S. 2005, A\&A, 431, 391

Zaitsev, V. V., \& Stepanov, A. V. 1982, Sov. Astron. Lett., 8, 132

Zimovets, I. V., \& Struminsky, A. B. 2010, Sol. Phys., 263, 163 